\title{
Estimativa de carbono individual para Araucaria angustifolia ${ }^{1}$
}

\author{
Carlos Roberto Sanquetta ${ }^{2}$, Ana Paula Dalla Corte ${ }^{2}$, \\ Francelo Mognon ${ }^{2}$, Greyce Charllyne Benedet Maas $^{2}$, Aurélio Lourenço Rodrigues ${ }^{2}$
}

\begin{abstract}
Individual carbon estimation for Araucaria angustifolia

Forests are important carbon sinks that contribute to climate change mitigation. Quantifying the carbon stock is critical for measuring such mitigation potential. Araucaria angustifolia (Bert.) O. Ktze. Araucariaceae is a key forest species in southern Brazil, due to its ecological and economic importance. This study aimed at comparing two procedures for estimating individual carbon stock $(C)$ of $A$. angustifolia, in pure stands established in the southern Paraná State, Brazil. Individual carbon stocks were determined for thirty trees, as well as correlations between $C$, dendometric variables, Biomass Expansion Factor $(B E F)$ and Root-to-Shoot Ratio $(R)$. Regression equations of $C$, concerning Diameter Breast Height $(D B H)$ and total height $(H)$, were adjusted and compared to trunk volume $(V)$ estimates combined with $B E F$ and $R$. $D B H$ and $H$ showed high correlation to $C$, what did not happen to tree age, $B E F$ and $R$. The regression equations of $C$, concerning $D B H$ and $H$, adjusted well to the data set, providing reliable estimates. The same happened to the volume equations combined with expansion factors, which also provided statistically acceptable estimates. No difference was observed between the two procedures tested, being both reliable for estimating the individual carbon stock.
\end{abstract}

KEY-WORDS: Araucaria angustifolia (Bert.) O. Ktze. Araucariaceae; biomass expansion factor; root-to-shoot ratio.

\section{INTRODUÇÃO}

As florestas exercem papel importante no balanço global de carbono, já que fixam carbono da atmosfera, mas, também, podem constituir-se em fonte de emissão (Sanquetta et al. 2011b). Por isto, exercem função fundamental nos esforços globais de combate a mudanças climáticas.

Quantificar o carbono em ecossistemas florestais e suas mudanças decorrentes de atividades humanas é o primeiro passo para melhor representar

\section{RESUMO}

As florestas são importantes sumidouros de carbono que contribuem para mitigar mudanças climáticas. Quantificar o estoque de carbono é fundamental para dimensionar este potencial de mitigação. Araucaria angustifolia (Bert.) O. Ktze. Araucariaceae é uma espécie florestal chave no sul do Brasil, devido à sua importância ecológica e econômica. Este estudo visou a analisar comparativamente dois procedimentos de estimativa do estoque individual de carbono $(C)$ de $A$. angustifolia, em povoamentos implantados no sul do Estado do Paraná. Foram determinados os estoques de carbono em trinta árvores, bem como as correlações entre $C$, variáveis dendrométricas, Fator de Expansão de Biomassa (FEB) e Razão de Raízes $(R)$. Equações de regressão de $C$, em função do Diâmetro à Altura do Peito $(D A P)$ e altura total $(H)$, foram ajustadas e comparadas com estimativas do volume do fuste $(V)$ combinadas com $F E B$ e $R$. $\mathrm{O} D A P$ e $H$ se correlacionaram significativamente $\operatorname{com} C$, o que não ocorreu com a idade, $F E B$ e R. As equações de regressão de $C$, em função de $D A P$ e $H$, se ajustaram bem aos dados e geraram estimativas acuradas. $\mathrm{O}$ mesmo ocorreu em relação às equações de volume, que, combinadas com os fatores de expansão, também propiciaram estimativas estatisticamente aceitáveis. Não houve diferença entre os dois procedimentos testados, sendo ambos acurados para estimar o estoque de carbono individual.

PALAVRAS-CHAVE: Araucaria angustifolia (Bert.) O. Ktze. Araucariaceae; fator de expansão de biomassa; razão de raízes.

as florestas na política climática, em escala regional, nacional e global (Watson 2009).

As estimativas de biomassa e carbono em florestas podem ser feitas por métodos diretos e indiretos (Sanquetta 2002). Os métodos diretos consistem em pesar toda a biomassa, geralmente por processo destrutivo. Esta abordagem é inviável para aplicação em grandes áreas, devido ao tempo e ao custo de execução. Por outro lado, os métodos indiretos implicam em emprego de algum tipo de modelagem da biomassa e do carbono, empregando-se fatores

1. Trabalho recebido em fev./2013 e aceito para publicação em jan./2014 (nº registro: PAT 22358).

2. Universidade Federal do Paraná (UFPR), Departamento de Ciências Florestais, Curitiba, PR, Brasil.

E-mails: carlos_sanquetta@hotmail.com, anapaulacorte@gmail.com, francelomognon@yahoo.com.br, greycemaas@yahoo.com.br, aurelio_lourenso@msn.com. 
e/ou equações. Entretanto, tais fatores ou equações precisam ser ajustados a uma base primária de dados, para garantir validade às estimativas.

Equações de regressão tendo o carbono $(C)$ como variável dependente e variáveis dendrométricas como independentes são, muitas vezes, chamadas de equações alométricas (Soares \& Tomé 2004). Elas são recomendáveis na maioria dos casos, pois oferecem predições diretas de $C$, em função das variáveis dendrométricas, e são de fácil medição e simples aplicação. Contudo, em termos de precisão e acurácia, nem sempre são satisfatórias, já que seus ajustes resultam em níveis de erro acima dos toleráveis em mensuração florestal, particularmente se a base de dados utilizada nos ajustes apresentar inconsistências.

Uma alternativa metodológica é o ajuste de equações de volume do fuste $(V)$, que é comumente estimado em inventários florestais convencionais e geralmente obtido com alto nível de precisão e acurácia, combinado com fatores que extrapolam a massa do fuste para toda a biomassa aérea e adicionalmente à biomassa subterrânea. Estes fatores são chamados de Fator de Expansão de Biomassa (FEB) e Razão de Raízes $(R)$ (Sanquetta et al. 2011a).

Segundo Soares \& Tomé (2004), equações alométricas são preferíveis para a estimativa de carbono florestal, mas, quando não disponíveis, pode-se lançar mão de fatores de expansão específicos para diferentes faixas etárias dos povoamentos florestais.

Este estudo objetivou comparar dois procedimentos usuais, na estimativa de carbono em povoamentos puros de Araucaria angustifolia Bert. O. Ktze. Araucariaceae.

\section{MATERIAL E MÉTODOS}

Os dados utilizados advêm de povoamentos puros implantados com Araucaria angustifolia Bert. O. Ktze. Araucariaceae, nos municípios de Quedas do Iguaçu e General Carneiro, sul do Estado do Paraná. O povoamento florestal de Quedas do Iguaçu $\left(25^{\circ} 20^{\prime} 58^{\prime}\right.$ 'S a $25^{\circ} 34$ ' $55^{\prime}$ 'S e $52^{\circ} 36^{\prime} 24^{\prime \prime} \mathrm{W}$ a $53^{\circ} 07^{\prime} 43^{\prime \prime} \mathrm{W}$ ) foi implantado nos anos de 1987 e 1988. Já o povoamento de General Carneiro (26 $26^{\circ} 35^{\prime \prime} \mathrm{S}$ a $26^{\circ} 26^{\prime} 13^{\prime \prime} \mathrm{S}$ e $51^{\circ} 19^{\prime} 49^{\prime \prime} \mathrm{W}$ a 51²5'29'”) foi implantado de 1969 a 1987.

Trinta árvores foram utilizadas para o presente estudo, as quais foram cortadas e pesadas pelo método direto, em 2003 e 2004. Este número foi definido admitindo-se, no máximo, $20 \%$ de erro na variável estoque individual de carbono. Foram determinadas as massas frescas em campo de toda a sua biomassa, separada em compartimentos (fuste sem casca, casca, galhada, folhagem e raízes). As raízes foram escavadas até a profundidade de $1,0 \mathrm{~m}$, lavadas e secas antes da pesagem, dentro da área de projeção da copa de cada indivíduo. Os dados de $D A P$ (diâmetro a 1,3 m do solo) e altura total $(H)$ das árvores também foram coletados. Amostras de 300-500 g de cada compartimento da biomassa foram retiradas para análise em laboratório.

As amostras de biomassa fresca retiradas em campo foram secas em estufa até massa constante $\left(70^{\circ} \mathrm{C}\right)$. Com base na relação entre massa fresca e seca, foram calculadas as massas secas de todos os compartimentos da biomassa levantados. Em seguida, as amostras foram trituradas para análise do teor de carbono ( $T$ ), empregando-se o método de combustão seca em câmara de infravermelho. Com base nos teores de carbono, foram calculados os estoques de carbono de todos os compartimentos da biomassa, que, somados, resultaram no estoque total individual de carbono $(C)$, variável principal desta pesquisa. As análises de laboratório foram realizadas ao longo do ano de 2004.

A partir da massa seca estimada para as árvores, foram calculados os Fatores de Expansão de Biomassa $(F E B)$ e as Razões de Raízes $(R)$ destas, utilizando-se as seguintes equações (IPCC 2006):

$$
F E B=\frac{M_{\text {copa }}+M_{\text {fuste }}}{M_{\text {fuste }}}=\frac{M_{\text {aérea }}}{M_{\text {fuste }}}
$$

$$
R=\frac{M_{\text {raizes }}}{M_{\text {aérea }}}
$$

em que $F E B=$ fator de expansão de biomassa (adimensional); $R$ = razão de raízes (adimensional); $M_{\text {copa }}=$ massa seca da copa da árvore $(\mathrm{kg}) ; M_{\text {fuste }}=$ massa seca do fuste da árvore $(\mathrm{kg}) ; M_{\text {aérea }}=$ massa seca do fuste da árvore + massa da copa da árvore $(\mathrm{kg})$; e $M_{\text {raizes }}=$ massa seca das raízes da árvore $(\mathrm{kg})$. Conceitualmente, o FEB é um fator que serve para extrapolar a massa do fuste da árvore para toda a biomassa aérea, considerando-se os galhos e a folhagem. Geralmente, é utilizado para cálculo do estoque de toda a biomassa aérea apenas com base no volume (usualmente estimado nos inventários florestais convencionais) e na densidade da madeira. 
Já a Razão de Raízes $(R)$ é um fator que expressa a razão da biomassa subterrânea pela biomassa aérea, servindo para calcular a massa de toda a biomassa da árvore.

Para a finalidade deste trabalho, foi determinado um valor médio de densidade básica da madeira de Araucaria angustifolia, fixado em $0,425 \mathrm{~g} \mathrm{~cm}^{-3}$ (Mattos et al. 2006). Este valor, que expressa a relação entre massa e volume, foi empregado para o cálculo do estoque individual de carbono, com base no volume do fuste e nos fatores de expansão (FEB e $R$ ).

Foram calculadas as estatísticas descritivas para as variáveis $D A P, H$, idade, $F E B, R$ e $C$ e analisadas as suas correlações, por meio de uma matriz de correlação linear simples de Pearson entre as mesmas.

Foram avaliados dois procedimentos para a estimativa do estoque individual de carbono $(C)$ : Estimativa do estoque individual de carbono a partir das equações de regressão - o $C$ foi estimado diretamente a partir de uma equação de regressão ajustada aos dados de campo, ou seja, em função de $D A P$ e $H$ (vide equações 4 a 7); e Estimativa do estoque individual de carbono a partir do volume do fuste $e$ dos fatores de expansão - feita a partir da seguinte expressão matemática, considerando-se que $V$ foi estimado com base nas equações 4 a 7 :

$C=V^{*} D A P * F E B *(1+R) * T$

Em ambas as abordagens, os modelos de regressão testados foram os seguintes:

Modelo 1: $\log Y=\beta_{0}+\beta_{1} * \log D A P$

Modelo 2: $Y=\beta_{0}+\beta_{1} * D A P^{2} * H$

Modelo 3: $\log Y=\beta_{0}+\beta_{1} * \log \left(D A P^{2} * H\right)$

Modelo 4: $\log Y=\beta_{0}+\beta_{1} * \log D A P+\beta_{2} * \log H$ em que $Y=$ estoque individual de carbono $(C)$ no procedimento 1 ou volume total do fuste $(V)$ no procedimento $2 ; D A P=$ diâmetro a $1,3 \mathrm{~m}$ do solo; $H=$ altura total; e $\beta_{i}=$ coeficientes dos modelos.

Uma equação, dentre as ajustadas, foi selecionada para as análises comparativas. Como critérios para seleção, foram utilizados o coeficiente de determinação ajustado $\left(\mathrm{R}^{2}{ }_{\text {ajust }}\right)$, erro padrão da estimativa absoluto $\left(\mathrm{S}_{\mathrm{yx}}\right)$ e em percentagem $\left(\mathrm{S}_{\mathrm{yx} \%}\right)$, assim como a análise gráfica dos resíduos.

Para a comparação das duas abordagens, na estimativa do estoque individual de carbono, utilizou-se o teste t pareado $(\alpha=0,05)$, confrontando o valor real de $C$ com suas estimativas $\hat{C}_{1}$ (regressão) e $\hat{C}_{2}$ (volume combinado com $F E B$ e $R$ ).

\section{RESULTADOS E DISCUSSÃO}

Para uma variação de 14 a 31 anos na idade das árvores, seus diâmetros variaram de $14,29 \mathrm{~cm}$ a $33,39 \mathrm{~cm}$ e suas alturas de $12,72 \mathrm{~m}$ a $19,90 \mathrm{~m}$. Em estoque individual de carbono, as variações foram de $25,91 \mathrm{~kg}$ a $228,43 \mathrm{~kg}$. O FEB variou de 1,05 a 2,06, indicando que existem árvores com pouca biomassa de copa e outras em que esta fração é maior que a do próprio fuste. $R$, por sua vez, variou de 0,03 a 0,12 , apontando que a participação da biomassa subterrânea também oscila de maneira expressiva entre indivíduos (Tabela 1).

Silveira (2010) destaca que trabalhos sobre o uso de $F E B$ para estimativa de carbono florestal são escassos no Brasil. O autor trabalhou com uma ampla gama de espécies nativas em Floresta Ombrófila Densa, no Estado de Santa Catarina.

Estudos sobre biomassa e carbono em A. angustifolia Bert. O. Ktze. Araucariaceae foram realizados por Sanquetta et al. (2003), Watzlawick (2003) e Schumacher et al. (2011), porém, sem avaliar o FEB e $R$. Deduzindo-se dos valores de

Tabela 1. Estatística descritiva para Diâmetro à Altura do Peito $(D A P)$, altura total $(H)$, idade, Fator de Expansão de Biomassa $(F E B)$, Razão de Raízes $(R)$ e estoque individual de carbono $(C)$, para povoamento de Araucaria angustifolia Bert. O. Ktze. Araucariaceae (Quedas do Iguaçu e General Carneiro, PR, 2003-2004).

\begin{tabular}{|c|c|c|c|c|c|c|}
\hline & $D A P$ & $H$ & Idade & \multirow{2}{*}{$F E B$} & \multirow{2}{*}{$R$} & $C$ \\
\hline & $\mathrm{cm}$ & $\mathrm{m}$ & anos & & & $\mathrm{kg}$ \\
\hline Média & 24,69 & 16,69 & 21,67 & 1,39 & 0,07 & 113,01 \\
\hline Mínimo & 14,29 & 12,72 & 14,00 & 1,05 & 0,03 & 25,91 \\
\hline Máximo & 33,39 & 19,90 & 31,00 & 2,06 & 0,12 & 228,43 \\
\hline Desvio padrão & 5,62 & 2,07 & 5,85 & 0,25 & 0,02 & 58,20 \\
\hline Coeficiente de variação & 22,76 & 12,39 & 27,00 & 17,94 & 31,15 & 51,50 \\
\hline
\end{tabular}


biomassa apresentados pelos autores, encontram-se valores médios respectivos de $F E B$ e $R$ de 1,34 e 0,18 , para povoamentos de 24 a 32 anos, em General Carneiro (Watzlawick 2003), de 1,32 e 0,15, para um povoamento de 27 anos, em Quedas do Iguaçu, em condições de corte raso (Schumacher et al. 2011), e de 1,39 para FEB, em General Carneiro, em plantações florestais com idades oscilando entre 14 e 32 anos (Sanquetta et al. 2003). Os valores de $F E B$ da literatura encontram-se dentro do espectro de valores observados neste estudo. Já os valores de $R$, nesta pesquisa, foram inferiores aos da literatura. Esta diferença pode ser atribuída à maior amplitude de idades deste estudo, em comparação com a da literatura mencionada.

Em povoamentos de Pinus implantados no Estado do Paraná, Sanquetta et al. (2011a) encontraram valores médios de $F E B$ e $R$ de 1,43 e 0,17 , respectivamente, com variações de 1,09 a 3,74 para $F E B$ e de 0,05 a 0,63 para $R$. Estudos sobre a proporção de biomassa em outras espécies são apresentados por Schneider et al. (2005), em Acacia mearnsii De Wild. Fabaceae, e Miranda et al. (2012), para Euterpe oleracea Mart. Arecaceae, entre outros.

A matriz de correlação simples entre as variáveis estudadas (Tabela 2) mostrou que aquela com maior correlação com o estoque de carbono total individual foi o $D A P$, seguida da altura total e da idade. Estas correlações foram positivas e significativas. Isto quer dizer que, quanto maior o porte das árvores e mais velhas forem, maior será seu estoque individual de carbono.

A correlação de $C$ com $F E B$ foi negativa e com $R$ positiva, mas não significativas. Isto quer dizer que os estoques individuais de carbono não são afetados pelas proporções em copa (galhada + folhagem) e

Tabela 2. Matriz de correlação simples entre as variáveis altura total $(H)$, idade, Fator de Expansão de Biomassa $(F E B)$, Razão de Raízes $(R)$ e estoque individual de carbono (C), para povoamento de Araucaria angustifolia Bert. O. Ktze. Araucariaceae (Quedas do Iguaçu e General Carneiro, PR, 2003-2004).

\begin{tabular}{lccrrrr}
\hline Variável & $D A P$ & $H$ & Idade & $F E B$ & $R$ & $C$ \\
\hline$D A P$ & 1,00 & & & & & \\
$H$ & $0,86^{*}$ & 1,00 & & & & \\
Idade & 0,57 & 0,63 & 1,00 & & & \\
$F E B$ & $-0,36$ & $-0,45$ & $-0,67$ & 1,00 & & \\
$R$ & $0,73^{*}$ & 0,55 & 0,38 & $-0,44$ & 1,00 & \\
$C$ & $0,92^{*}$ & $0,79 *$ & 0,65 & $-0,26$ & 0,54 & 1,00 \\
\hline * Significativo a $5 \%$. & & & & &
\end{tabular}

de biomassa subterrânea (raízes), no presente caso. $F E B$ e $R$ não possuem correlações significativas entre si. FEB mostrou correlação negativa com a idade e $R$ positiva com o $D A P$, o que significa que, com o avanço da idade, a proporção de copa tende a diminuir, e, com o aumento do porte das árvores em $D A P$, a participação relativa das raízes se torna maior.

Os dados apresentados por Watzlawick (2003) sugerem comportamento semelhante. Resultados para plantios de Pinus taeda L. Pinaceae, no Paraná, mostraram redução de $F E B$ e $R$ com a idade e com o DAP (Sanquetta et al. 2011a). Isto, possivelmente, decorreu da idade mínima considerada no presente estudo, que se inicia em 14 anos.

Ratuchne (2010), em trabalhos com Araucária, encontrou coeficientes de correlação da biomassa total individual com variáveis dendrométricas comparáveis aos do presente estudo, mas inferiores aos aqui apresentados. Isto decorre da gama de espécies analisadas pelo autor.

As equações de carbono ajustadas apresentaram coeficientes de determinação de 0,85 a 0,86 e erros padrões da estimativa próximos a $20 \%$. Dentre os modelos testados, o de número 1 foi o que propiciou estimativas mais precisas, embora todos os quatro possam ser considerados satisfatórios (Tabela 3).

Schumacher et al. (2011) obtiveram valores de $\mathrm{R}_{\text {ajust }}^{2}$ superiores e $\mathrm{S}_{\mathrm{yx} \%}$ inferiores, em modelagem da biomassa total individual de Araucaria angustifolia Bert. O. Ktze. Araucariaceae. Isto decorreu do fato de os autores utilizarem apenas dados de uma única idade (27 anos). Sanquetta et al. (2003) encontraram ajustes satisfatórios para a biomassa do fuste, em função de $D A P$ e $H$, com erros padrões da estimativa baixos e

Tabela 3. Ajuste das equações para estimar o estoque de carbono total para Araucaria angustifolia Bert. O. Ktze. Araucariaceae (Quedas do Iguaçu e General Carneiro, PR, 2003-2004).

\begin{tabular}{ccccccc}
\hline Modelo & $\beta_{0}$ & $\beta_{1}$ & $\beta_{2}$ & $\mathrm{R}_{\text {ajust }}^{2}$ & $\mathrm{~S}_{\mathrm{yx}}$ & $\mathrm{S}_{\mathrm{yx} \%}$ \\
\hline \multicolumn{7}{c}{ Carbono } \\
1 & $-1,2538$ & 2,3474 & - & 0,86 & 21,70 & $19,20^{*}$ \\
2 & 7,2153 & 0,0095 & - & 0,85 & 22,76 & 20,14 \\
3 & $-1,7967$ & 0,9506 & - & 0,85 & 22,80 & 20,18 \\
4 & $-1,5232$ & 2,1400 & 0,4559 & 0,86 & 22,32 & 19,75 \\
\hline \multicolumn{7}{c}{ Volume } \\
1 & $-3,7737$ & 2,4515 & - & 0,96 & 0,07 & 18,73 \\
2 & 0,00025 & 0,00004 & - & 0,99 & 0,04 & $9,36^{*}$ \\
3 & $-3,8757$ & 0,8665 & - & 0,94 & 0,09 & 22,51 \\
4 & $-3,8483$ & 1,9593 & 0,5979 & 0,95 & 0,08 & 21,01 \\
\hline * Equações de melhor ajuste em função do maior $\mathrm{R}_{\text {ajust }}^{2}$ e menor $\mathrm{S}_{\mathrm{yx} \%}$ \\
\multicolumn{7}{c}{}
\end{tabular}


coeficientes de determinação altos, porém, insatisfatórios para as biomassas parciais dos compartimentos.

As equações de volume apresentaram coeficientes de determinação de 0,94 a 0,99 , com erros padrões da estimativa variando de $9 \%$ a $22 \%$. O modelo que apresentou maior $\mathrm{R}_{\text {ajust }}^{2}$ e menor $\mathrm{S}_{\mathrm{yx} \%}$ foi o de número 2 (Tabela 3 ). Os resíduos dos modelos de carbono e de volume com melhor desempenho são destacados na Figura 1.

Vários autores têm desenvolvido estudos sobre ajuste de equações de volume para a espécie em estudo (Schneider \& Finger 2000, Mattos et al. 2010, Oliveira et al.2011). Na maioria dos casos, os ajustes são satisfatórios e comparáveis aos deste estudo, principalmente devido à forma regular do fuste da espécie.

Analisando-se as estimativas de estoque individual de carbono para a espécie em estudo, com a melhor equação de regressão $\left(\hat{C}_{l}\right)$ e os respectivos valores reais desta variável, verificou-se que a diferença média foi de $-1,22 \%$, ou seja, o valor médio real foi de $113,01 \mathrm{~kg}$ e o estimado de $111,63 \mathrm{~kg}$ $(\mathrm{p}=0,7248)$. Quando se compararam as estimativas com a equação $3\left(\hat{C}_{2}\right)$, que se baseia na combinação da

Modelo 1

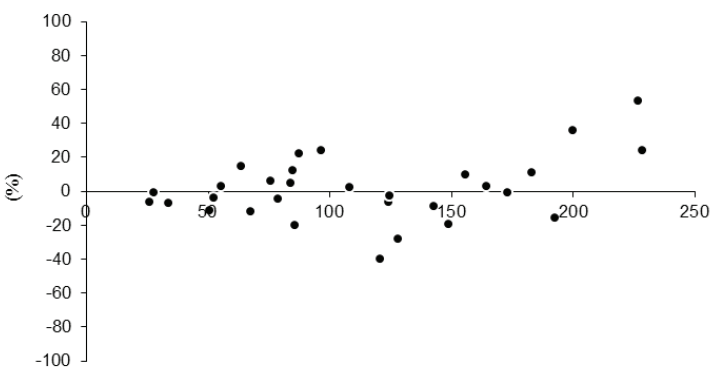

$\mathrm{C}(\mathrm{kg})$

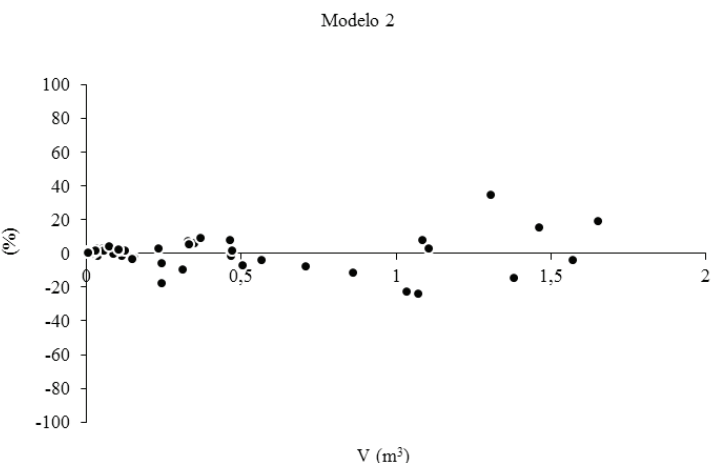

Figura 1. Distribuição de resíduos das equações de carbono (a) e de volume (b) de melhor desempenho para Araucaria angustifolia Bert. O. Ktze. Araucariaceae (Quedas do Iguaçu e General Carneiro, PR, 2003-2004).

(b) estimativa de volume (no presente caso, o modelo 2 foi utilizado, devido à sua melhor performance) e dos fatores de expansão (FEB e $R$ ) e os respectivos valores reais de $C$, verificou-se diferença média de $5,28 \%$, com valor estimado médio de $118,98 \mathrm{~kg}$ $(\mathrm{p}=0,0859)$.

Essas diferenças, quando analisadas pelo teste $\mathrm{t}$ pareado $(\mathrm{p}<0,05)$, foram consideradas não significativas, ou seja, ambas as estimativas $\left(\hat{C}_{1}\right.$ e $\left.\hat{C}_{2}\right)$ não foram estatisticamente diferentes do valor real de $C$. Na Figura 2, pode-se visualizar a relação dos valores reais de $C$ e suas estimativas com a equação de regressão de carbono e a equação de volume combinada com os fatores de expansão.

A obtenção do estoque de carbono florestal não é factível por procedimentos diretos. Por isto, é importante se valer das relações entre as dimensões das árvores para, a partir das variáveis de fácil obtenção, como o diâmetro, a altura e a idade, realizar mais prontamente as estimativas pelos métodos indiretos.

Existem várias maneiras de se estimar o estoque de carbono em árvores, porém, as mais usuais são a utilização de equações alométricas e a aplicação de fatores de expansão (Soares \& Tomé 2004). Segundo estes autores, o uso de equações alométricas para estimativa de biomassa é preferível, mas, quando não disponíveis, pode-se utilizar fatores de expansão variáveis para diferentes idades.

Sanquetta et al. (2011b) encontraram uma clara relação de dependência de $F E B$ e $R$ com a idade, mostrando tendências curvilíneas negativas para Pinus, no Estado do Paraná. Resultados semelhantes

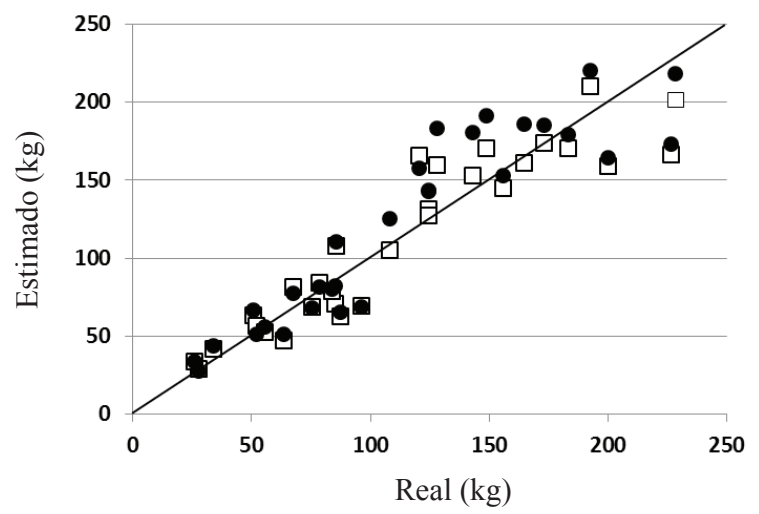

Figura 2. Valores estimados versus reais, para os dois procedimentos de estimativa individual de carbono, em Araucaria angustifolia Bert. O. Ktze. Araucariaceae (Quedas do Iguaçu e General Carneiro, PR, 2003-2004). 
para FEB foram obtidos por Soares \& Tomé (2004), em Eucalyptus globulus Labill. Myrtaceae, embora o conceito desta grandeza empregado por tais autores tenha sido diferente (biomassa aérea total dividida pelo volume do tronco).

$F E B$ e $R$, devido à sua simplicidade e aplicação imediata, vêm sendo empregados alternativamente para estimar a biomassa e, por consequência, o carbono. Representam uma maneira simples de extrapolar o carbono do fuste (geralmente mais facilmente estimado e possível de se obter pela multiplicação do volume pela densidade da madeira) para toda a biomassa viva da árvore.

Normalmente, $F E B$ e $R$ são assumidos como constantes, o que, nem sempre, é o caso. O próprio Painel Intergovernamental para as Mudanças Climáticas publica tabelas com valores médios (default)

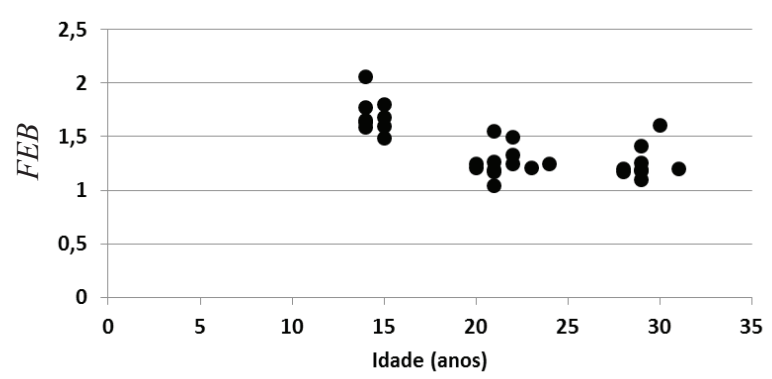

(a)

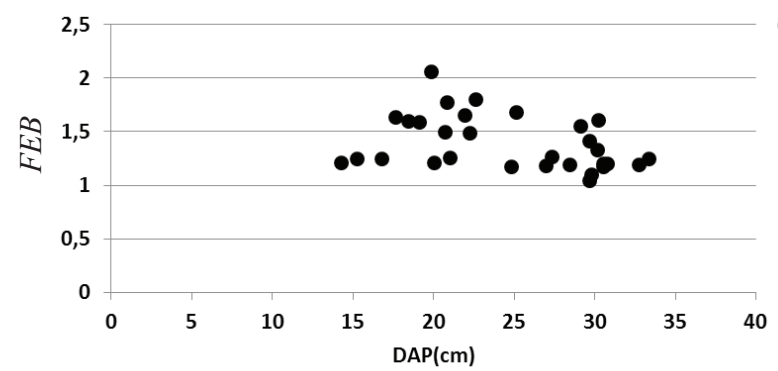

(c)

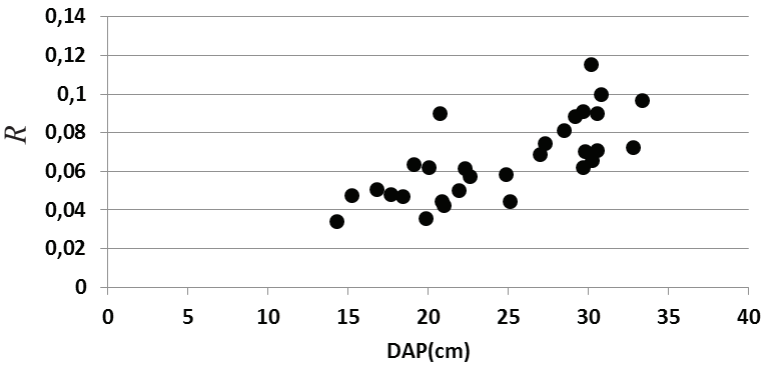

(d)

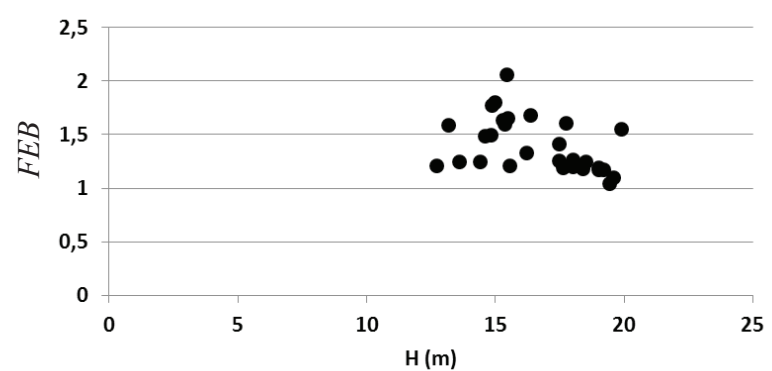

(e)

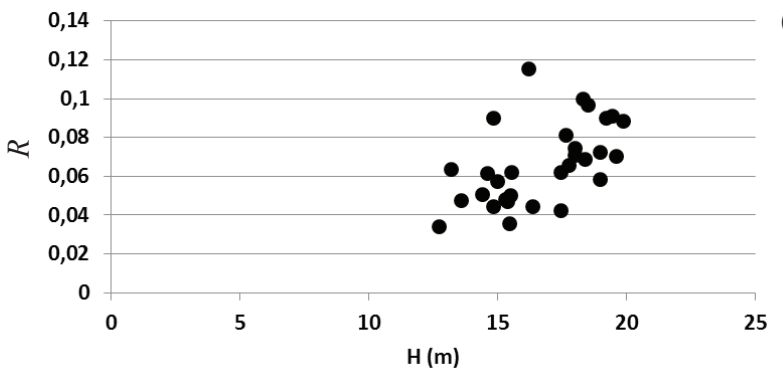

Figura 3. Relação de dependência de $F E B$ e $R$ com o tamanho e a idade, em Araucaria angustifolia Bert. O. Ktze. Araucariaceae: $F E B \times$ idade (a); $R$ x idade (b); $F E B \times D A P($ c) $R \times D A P($ d) $; F E B \times H$ (e); e $R \times H$ (f) (Quedas do Iguaçu e General Carneiro, PR, 2003-2004).

para várias florestas do mundo (IPCC 2013), porque faltam pesquisas sobre $F E B$ e $R$ para a maior parte das florestas do mundo, especialmente nos trópicos. O uso de valores default (médios) poderia representar problemas nas estimativas de biomassa e carbono, mas, muitas vezes, é a única alternativa, pois, em muitos casos, não existem equações alométricas disponíveis. Os dois projetos florestais de Mecanismo de Desenvolvimento Limpo (MDL) do Brasil, registrados nas Nações Unidas e elaborados pelas empresas Plantar e AES Tietê (UNFCCC 2013), lançam mão do uso de valores fixos de $F E B$ e $R$, na estimativa das emissões certificadas de redução de $\mathrm{CO}_{2}$.

No presente estudo, as estimativas pelos dois procedimentos se mostraram muito próximas. Isto deveu-se à inexistência de uma relação clara de dependência de $F E B$ e $R$ com a idade (Figura 3), ou com

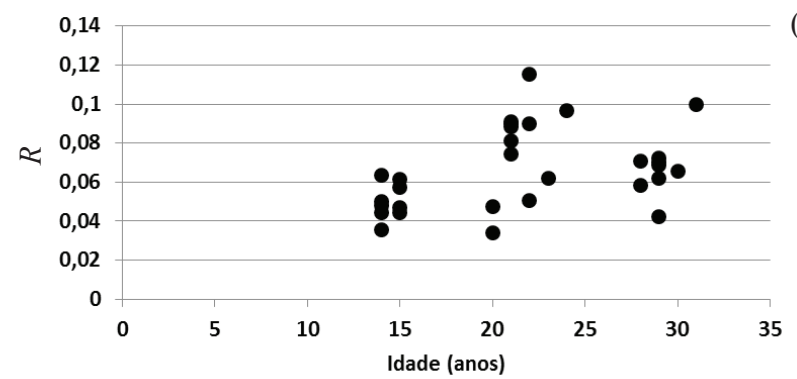


as variáveis dendrométricas $D A P$ e $H$, ao contrário do observado em outros estudos previamente citados.

As argumentações de Soares \& Tomé (2004) são pertinentes, quando se consideram povoamentos com grandes amplitudes etárias. Todavia, é importante observar que, nas idades mais jovens, ocorrem as maiores variações de $F E B$ e $R$, devido às taxas de crescimento mais aceleradas e à diferença na alocação da biomassa aos distintos órgãos, com a maturação da planta.

Por outro lado, em povoamentos com idades intermediárias a avançadas, que apresentam taxas de crescimento tendendo à estabilização e uma definição arquitetônica da planta, existe uma tendência de $F E B$ e $R$ não se alterarem mais com o avanço da idade (como se pode verificar nos trabalhos retrocitados). Neste caso, a suposta vantagem das equações alométricas não se materializa, o que ocorreu neste estudo, o qual demonstrou não existir diferença estatística entre os dois procedimentos, para estimar o estoque individual de carbono em Araucaria angustifolia Bert. O. Ktze. Araucariaceae.

Por fim, é relevante salientar que estimativas de volume são sempre mais precisas e acuradas que estimativas de carbono. Por isto, uma possível vantagem do procedimento poderia emergir das discussões deste trabalho. Se não existirem relações de dependência da variável carbono com a idade e o porte das árvores ( $D A P$ e $H)$, é possível obter ganhos estatísticos com o segundo procedimento de estimativa de carbono aqui apresentado, além do fator simplicidade. A influência da densidade da madeira, neste contexto, precisa ser mais detalhadamente explorada.

\section{CONCLUSÕES}

1. As variáveis dendrométricas $D A P$ e altura exprimiram, significativamente, as variações no estoque individual de carbono $(C)$ da espécie analisada e nas condições vigentes no estudo. Isto não ocorreu com a idade, que apresentou correlação não significativa com $C$, o mesmo ocorrendo com os fatores de expansão $F E B$ e $R$.

2. Equações de carbono ajustadas por regressão direta do estoque individual de carbono, em função das variáveis dendrométricas, geraram estimativas satisfatórias. $\mathrm{O}$ mesmo pode ser dito em relação às equações de volume, que, combinadas com os fatores de expansão, também propiciaram estima- tivas aceitáveis e não diferentes, estatisticamente, dos valores reais.

3. Não houve diferença entre os dois procedimentos testados, a saber: equações de regressão versus combinação de equações de volume e fatores de expansão. Possivelmente, isto tenha decorrido do fato de não haver uma relação de dependência de $F E B$ e $R$ com a idade.

\section{REFERÊNCIAS}

INTERGOVERNMENTAL PANEL ON CLIMATE CHANGE (IPCC). Guidelines for national greenhouse gas inventories: good practice guidance. 2006. Disponível em: $<$ http://www.ipcc.ch>. Acesso em: 11 jan. 2013.

INTERGOVERNMENTAL PANEL ON CLIMATE CHANGE (IPCC). Emissions factor database. 2013. Disponível em: <http://www.ipcc.ch>. Acesso em: 11 jan. 2013.

MATTOS, P. P. de et al. Caracterização física, química e anatômica da madeira de Araucaria angustifolia (Bert.) O. Kuntze. Colombo: Embrapa, 2006. (Comunicado técnico, 160).

MATTOS, P. P. de et al. Equação de volume para araucárias centenárias na Reserva Florestal da Embrapal Epagri. Colombo: Embrapa, 2010. (Comunicado técnico, 256).

MIRANDA, D. L. C. et al. Biomassa e carbono em Euterpe oleracea Mart., na Ilha do Marajó - PA. Floresta e Ambiente, Seropédica, v. 19, n. 3, p. 336-343, 2012.

OLIVEIRA, M. F. de et al. Equação de volume para um plantio de Araucaria angustifolia em Rio Negro, PR. Colombo: Embrapa, 2011. (Comunicado técnico, 275).

RATUCHNE, L. C. Equações alométricas para estimativa de biomassa, carbono e nutrientes em uma Floresta Ombrófila Mista. 2010. 111 f. Dissertação (Mestrado em Agronomia) - Universidade Estadual do Centro-Oeste, Guarapuava, 2010.

SANQUETTA, C. R. Metodologias para determinação de biomassa florestal. In: SANQUETTA, C. R. et al. (Eds.). As florestas e o carbono. Curitiba: UFPR, 2002. p. 119-140.

SANQUETTA, C. R.; DALLA CORTE, A. P.; SILVA, F. Biomass expansion factor and root-to-shoot ratio for Pinus in Brazil. Carbon Balance and Management, London, v. 6, n. 6, p. 1-8, 2011a.

SANQUETTA, C. R.; DALLA CORTE, A. P.; MAAS, G. C. B. The role of forests in climate change. Quebracho, Santiago del Estero, v. 19, n. 1/2, p. 84-96, 2011 b. 
SANQUETTA, C. R. et al. Relações individuais de biomassa e conteúdo de carbono em plantações de Araucaria angustifolia e Pinus taeda no sul do Estado do Paraná, Brasil. Acadêmica: Ciências Agrárias e Ambientais, Curitiba, v. 1, n. 3, p. 33-40, 2003.

SCHNEIDER, P. R.; FINGER, C.A. G. Manejo sustentado de florestas inequiâneas heterogêneas. Santa Maria: UFSM, 2000.

SCHNEIDER. P. R. et al. Determinação indireta do estoque de biomassa e carbono em povoamentos de acácianegra (Acacia mearnsii De Wild.). Ciência Florestal, Santa Maria, v. 15, n. 4, p. 391-402, 2005.

SCHUMACHER, M. V. et al. Produção de biomassa no corte raso em plantio de Araucaria angustifolia (Bertol.) Kuntze de 27 anos de idade em Quedas do Iguaçu, PR. Ciência Florestal, Santa Maria, v. 21, n. 1, p. 53-62, 2011.

SILVEIRA, P. Estimativa da biomassa e carbono acima do solo em um fragmento de Floresta Ombrófila Densa utilizando o método da derivação do volume comercial. Floresta, Curitiba, v. 40, n. 4, p. 789-800, 2010.

SOARES, P.; TOMÉ, M. Analysis of the effectiveness of biomass expansion factors to estimate stand biomass. In: HASENAUER, H.; MAKELA, A. (Eds.). Modeling forest production. Viena: BOKU University of Natural Resources and Applied Life Sciences, 2004. p. 368-374.

UNITED NATIONS FRAMEWORK CONVENTION ON CLIMATE CHANGE (UNFCCC). Clean development mechanism. 2013. Disponível em: <http://www.unfccc. int>. Acesso em: 11 jan. 2013.

WATSON, C. Forest carbon accounting: overview and principles. London: UNDP/UNEP, 2009.

WATZLAWCK, L. F. Estimativa de biomassa e carbono em Floresta Ombrófila Mista e plantações florestais a partir de dados de imagens do satélite IKONOS II. 2003. 120 f. Tese (Doutorado em Engenharia Florestal) Universidade Federal do Paraná, Curitiba, 2003. 